\title{
COMPARATIVE STUDY OF LUNAR ROUGHNESS FROM MULTI - SOURCE DATA
}

\author{
Y. Lou, Z. Kang \\ School of Land Science and Technology, China University of Geosciences(Beijing), 29 Xueyuan Road, Haidian \\ District, Beijing 100083, China - aileenlou@163.com
}

KEY WORDS: Lunar, roughness, Root-mean-square Height, Morphological Surface Roughness, DEM, DOM

\begin{abstract}
:
The lunar terrain can show its collision and volcanic history. The lunar surface roughness can give a deep indication of the effects of lunar surface magma, sedimentation and uplift. This paper aims to get different information from the roughness through different data sources. Besides introducing the classical Root-mean-square height method and Morphological Surface Roughness (MSR) algorithm, this paper takes the area of the Jurassic mountain uplift in the Sinus Iridum and the Plato Crater area as experimental areas. And then make the comparison and contrast of the lunar roughness derived from LRO's DEM and CE-2 DOM. The experimental results show that the roughness obtained by the traditional roughness calculation method reflect the ups and downs of the topography, while the results obtained by morphological surface roughness algorithm show the smoothness of the lunar surface. So, we can first use the surface fluctuation situation derived from RMSH to select the landing area range which ensures the lands are gentle. Then the morphological results determine whether the landing area is suitable for the detector walking and observing. The results obtained at two different scales provide a more complete evaluation system for selecting the landing site of the lunar probe.
\end{abstract}

\section{INTRODUCTION}

Because of long time in the lunar geologic age, the moon is relatively cold, rigid and complete. And the surface has not been affected by plate movement, atmosphere, water or life, so the moon keeps the geological record for nearly 4 billion years (Jolliff B L,2006). The surface roughness is the ruggedness in the meaning of topography. In a certain research scope, it refers to a mathematical expression of the surface fluctuation condition at an analysis scale. Meantime it can reflect the rolling extent of surface. The roughness records the geologic activities such as erosion, sedimentation, accumulation and filling of the planets. And it is one of the important parameters to evaluate the safety of ground engineering. At the same time, it can also provide an important reference for finding a soft landing field of the surveyed spacecraft in the appropriate terrain (Shepard M $\mathrm{K}, 2001$ ). As a result of the sun weathered, the migration of moon shell by the internal stress, and ups and downs on the surface of the moon by the external impact, the trances generated on the lunar surface is the roughness. So it is possible to analyse the evolution history, the internal stress and the external impact of the lunar according to the roughness.

For the calculation of the lunar surface roughness, Michael K. Shepard proposes Root-mean-square height, Root-mean-square deviation, Root-mean-square slope, Autocorrelation length, Median and absolute slope and some other methods (Shepard M $\mathrm{K}, 2001)$. Many domestic and foreign scholars also use the Hurst index as a measurement of the lunar roughness (Orosei, $\mathrm{R}$ et $\mathrm{al}, 2003)$. These above methods are suitable for data with terrain elevation values. For grayscale binary images, in recent years $\mathrm{Cao}$, et al. proposes a morphological algorithm to calculate the lunar roughness (W. Cao et al,2014). The roughness of three specific lunar surface highlands has been studied, and the result shows the roughness plays an important role in studying the material composition of the lunar surface and the geological age of different stratigraphic units (Yokota Y et al.2008). The roughness of Mare Imbrium proves that there is a correlation between the lunar roughness and the lithology of the geological units (Yan Yanzi et al, 2014). The surface roughness of each parameter in the horizontal section of Sinus Iridum has been calculated, and the geomorphological features of the area are interpreted (Xi Xiaoxun et al, 2012). Few people use multisource data to compare and analyse the old and new methods to searching new information.

In this paper, we selected the tail of Montes Jura in the eastern of Sinus Iridum and the Crater Plato as the study areas. Based on the Digital Elevation Model of LRO and the Digital Orthophoto Map of Chang'e II, the roughness is calculated respectively by Root-mean-square height and Morphological Surface Roughness algorithm. Then we compare the two different results to obtain the characteristics and application scope of the two roughness algorithms. It can be more conducive to select the appropriate landing point for the lunar probe in the future.

\section{DATA AND METHOD}

\subsection{Research area}

In this paper, Sinus Iridum and Crater Plato the two large craters, which are created by "Heavy Bombardment" in Mare Imbrium, are selected as the experimental areas. The whole area is filled with mare basalt after the heavy impact.

Sinus Iridum is an important bay in the northwest of Mare Imbrium with a central latitude and longitude of N44 ${ }^{\circ} 6$ ', W $31^{\circ} 30$ ', a diameter of $259 \mathrm{~km}$ and a bottom area of 47750.927 $\mathrm{km}^{2}$. The northwest of Sinus Iridum is surrounded by the Montes Jura and is adjacent to the craters of crater Bianchini and crater Maupertuis (Chen Shenbo, et al,2010). And it is the landing point for CE-3 satellite. This paper focuses on the tail of the southeast of Montes Jura which is covered by various topography, such as plains, mountains and impact craters. This area is mainly covered by ridges. And some sporadic small ejecta are also scattered. So the various geometric roughness features can be comprehensive analysed.

Crater Plato is a large pit located between the north of Mare Imbrium and Mare Frigoris, and its west is Montes Jura and Sinus Iridum (John W M G, 1972). The center position is about N51 ${ }^{\circ}$ 6 ', W9 ${ }^{\circ} 5^{\prime}$. (USGS, 2008). The crater is an irregularly polygonal, with a diameter of about $106 \mathrm{~km}$ and an average crater depth of 
$1.8 \mathrm{~km}$. It is now widely believed that the Crater Plato is generated after Mare Imbrium event, and the crater age is about $3.84 \mathrm{Ga}$. The bottom of crater is full of basal.

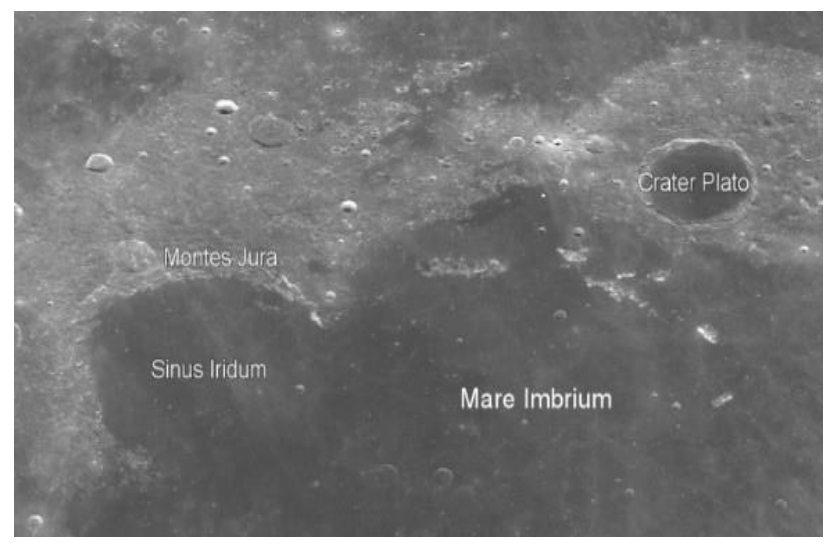

Figure 1. DOM of the experimental area

\subsection{Roughness calculation Based on DEM}

\subsubsection{Data}

The data used in this study is DEM whose accuracy is $30 \mathrm{~m}$ from LRO (Lunar Reconnaissance Orbiter). LRO is the first mission of the National Aeronautical and Space Pioneer Robot Program, which was implemented on June 18, 2009. LOLA is the laser altimeter of the six scientific instruments (Smith, D.et al,2010a; Smith, D.et al,2010b) . LOLA has 5 beams, with a nominal accuracy of $10 \mathrm{~cm}$. It can be used to characterize the microgeomorphic features on the lunar surface and select the future landing point for robots and human beings (Rosenburg et al, 2011). Using the global elevation data obtained by the sensor, lunar surface DEM is produced. The three-dimensional rendering images of the experimental area DEM as shown below.

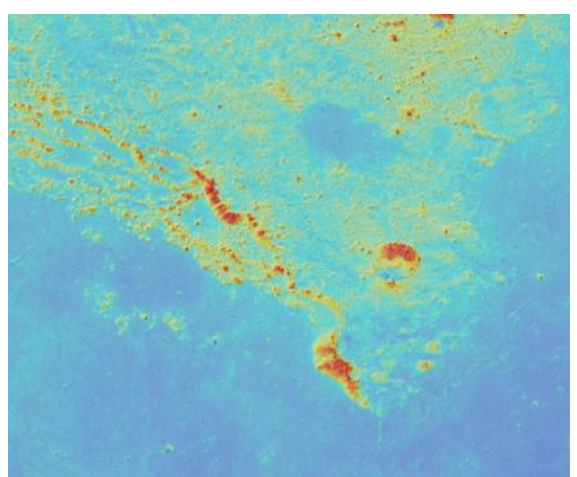

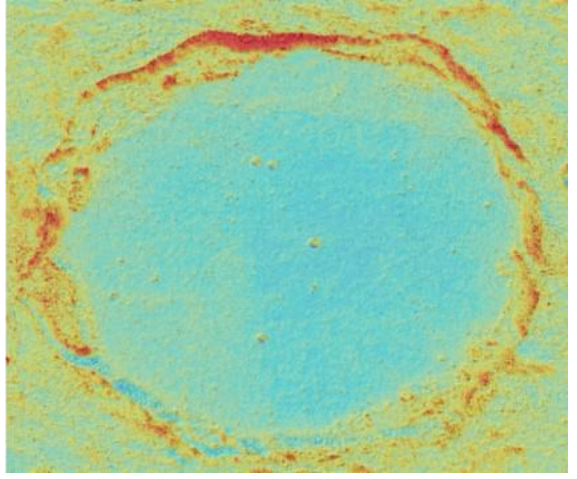

Figure 2. DEM Height rendering images of the tail of Montes Jura southeast (up) and Crater Plato (down)

\subsubsection{Root-mean-square height}

Shepard et al. have proposed several parameters for quantifying the planet's surface roughness. These parameters are usually defined on the basis of elevation data. The relatively commonly used and simple ones are the Root-mean-square height and Rootmean-square deviation. The Root-mean-square height shows the extent of the surface height deviating from the average height, which is expressed in the vertical direction. While the Rootmean-square deviation shows the change in the horizontal height which uses the structural function. The meaning of the two method is more or less the same, so this paper selects the Rootmean-square height for roughness calculation.

In general, a best fit linear function is subtracted from the DEM data. Through simplifying, a series of height values with the zero average are gotten. Expressed as

$\xi=\left[\frac{1}{n-1} \sum_{i=1}^{n}\left(z\left(x_{i}\right)-\bar{z}\right)^{2}\right]^{1 / 2}$

where $\mathrm{n}$ is the number of sample points

$\mathrm{z}\left(x_{i}\right)$ is the height of the point $x_{i}$ in the lunar surface $\bar{z}$ is the average of all elevation

In this paper, the raster image is read line by line to sample. And the $3 * 3$ window is used to calculate the whole DEM data. The average value of all the pixels in the window is calculated. Then the formula (1) is used to calculate the Root-mean-square height value of the central pixel to replace its original value.

\subsubsection{Results}

The roughness based on the two experimental DEM regions is calculated by the Root-mean-square height, and the results are shown in Fig.3. It can be seen that the result derived from the traditional method expresses the extent of the terrain ups and downs. The lunar roughness shows the dichotomy characteristic. That is, the lunar mare roughness is low, while roughness of the highland is high. The higher values of roughness are mainly distributed at the edge of the craters, which own the changeable terrain. While the lower roughness is mainly distributed in plains, because the terrain is gentle and the terrestrial changes are small.

The roughness both in the ridges and the fissures is high in the Montes Jura region. The mountain area is not all covered by the high roughness, there are also some gentle areas that own low 
roughness among the mountains. The roughness of Crater Plato indicates that there is a clear boundary between the bottom area and the edge. And the boundary between the ejecta and the edge is also clear. The bottom, the wall, the edge and the ejecta can be clearly distinguished by using the roughness image. And it can be seen that some sporadic small impact craters exist at the bottom with a large rough value.
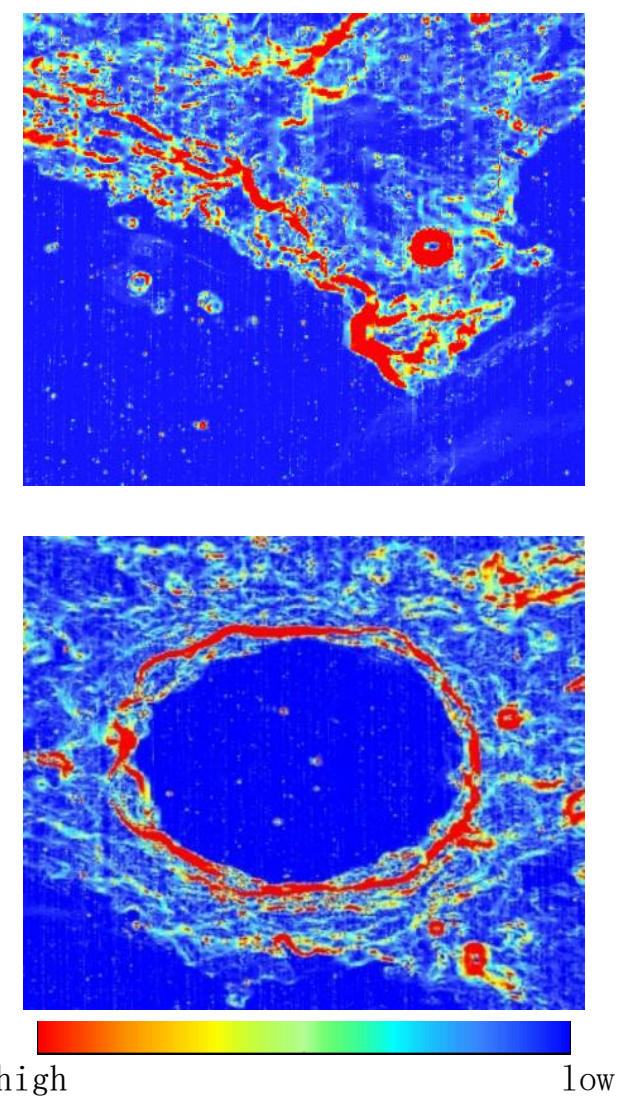

Figure 3. RMSH results images of the tail of Montes Jura southeast (up) and Crater Plato (down)

\subsection{Roughness calculation Based on DOM}

\subsubsection{Data}

CE-2 satellite successfully launched on October 1, 2010. Besides six goals had been successful completed, a number of important scientific data had gotten until April 1, 2011. CE-2 loads 5 categories of scientific detection equipment: CCD stereo camera, laser altimeter, $\gamma / \mathrm{X}$-ray spectrometer, microwave detectors and space environment detector. During the half year flighting around the moon, the CCD stereo camera takes photos of the global surface to obtain the lunar digital orthophoto map with a spatial resolution of $7 \mathrm{~m}$ (Ye J et al. 2013). The CE-2 DOM of the two experimental areas are shown as Figure 4.
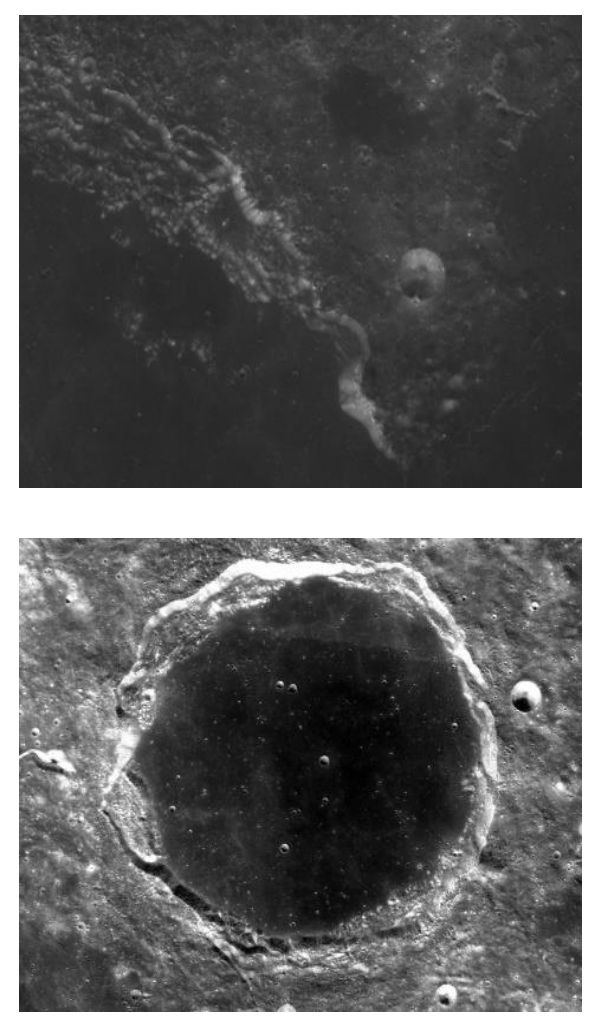

Figure 4. CE-2 DOM of the tail of Montes Jura southeast (up) and Crater Plato (down)

\subsubsection{Morphological Surface Roughness}

In this paper, we use the Morphological Surface Roughness proposed by W. Cao to deal with the DOM of CE-2. Unlike traditional roughness calculations based on ground elevation values, the algorithm utilizes grey-scale images. The surface roughness of the grey-scale images is defined as the difference between Morphological Closing operator and Morphological Opening operator. And it is usually constructed by the highest and lowest points of the structuring element's (SE) shape (P. Soille, 2013; W.Gonzalez and RE Woods, 2013). As an important theory of geological applications, Solide puts forward two common morphological operators: morphological opening and morphological closing (P. Soille, 2013). MO operator removes the redundant structures created by erosion. The MO function $\gamma$ is defined as follows:

$$
\left[\gamma_{B}(f)\right](x)=\delta_{B}\left[\varepsilon_{B}(f)\right]
$$

MC solves the problem of dilation by implementing erosion in the dilated surface.MC function $\emptyset$ is defined as

$$
\left[\emptyset_{B}(f)\right](x)=\varepsilon_{B}\left[\delta_{B}(f)\right]
$$

$$
\text { Where } \quad \begin{aligned}
{\left[\varepsilon_{B}(f)\right](x) } & =\min _{b \in B} f(x+b) \\
{\left[\delta_{B}(f)\right](x) } & =\max _{b \in B} f(x+b)
\end{aligned}
$$

Using the two operations two types of roughness forms can be 
produced. MO operation extracts the convex area while MC represents roughness characteristics by the concave distribution. The difference between the two surfaces is defined as the terrain surface roughness, the formula is as follows:

$$
R_{M S R}=\emptyset_{B}(f)-\gamma_{B}(f)
$$

\subsubsection{Results}

The roughness results obtained by using the MSR algorithm are shown as figure 5 . We can know that the roughness obtained by MSR is described on a smaller scale, which is not related much to the overall fluctuation of the area. It is a description of the roughness in a small range. Except a crater shows high roughness, the roughness value of Montes Jura other regions is low. This area is in a steady condition. In spite of that, the roughness difference between mountains and plains can also be clearly distinguished. However, the roughness at the bottom of the crater with light fluctuation is high, and the ejecta around the crater has the high roughness. There are also some unusual low roughness points in the region, the following part will describe the reason in detail.
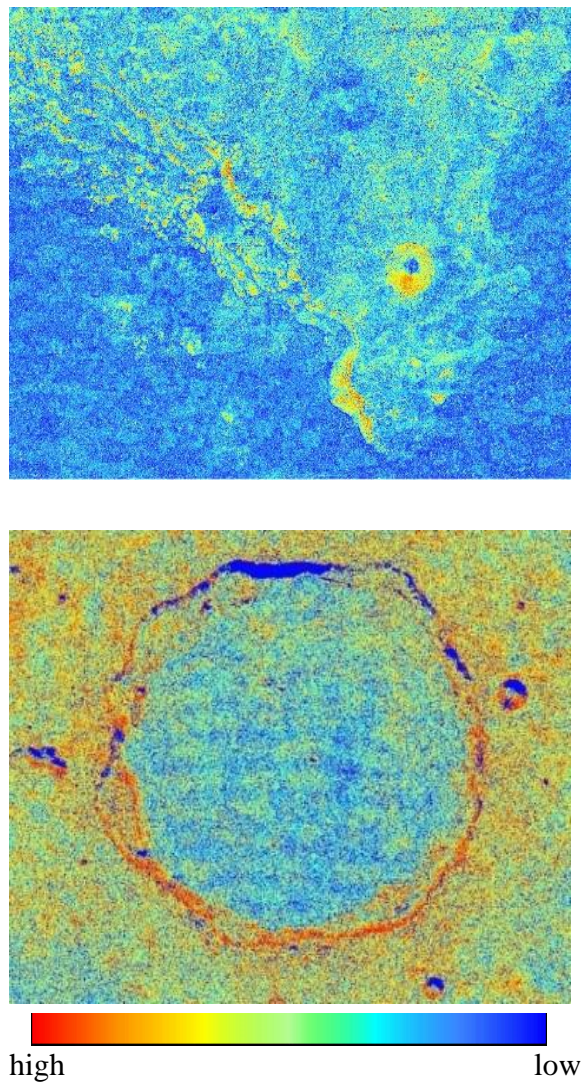

Figure 5. Results by using MSR of the tail of Montes Jura southeast (up) and Crater Plato (down)

\section{RESULTS AND DISCUSSION}

\subsection{Montes Jura}

The distribution of Montes Jura and every ridge can be clearly observed on the RMSH image. The roughness values of the mountain area are high, especially in the areas which the elevation of ridge is jumped. However, it is not obvious found on the MSR image. Compared with the lunar mare, the roughness of the mountain area is high, but the extent of roughness is not a lot Because the morphological algorithm eventually shows the smoothness of the surface. As long as the lithology of the region is the same one, the surface physical properties tend to be consistent, so the range of the roughness change is small. In addition, the roughness of lunar mare is low by using RMSH, while on the MSR image there are some high roughness areas existing on the surface because of the unsmooth basalt. The correlation between the lunar roughness and the lithology of the geological unit reflects the influence of the geological effect on the formation and evolution of the lunar landscape.
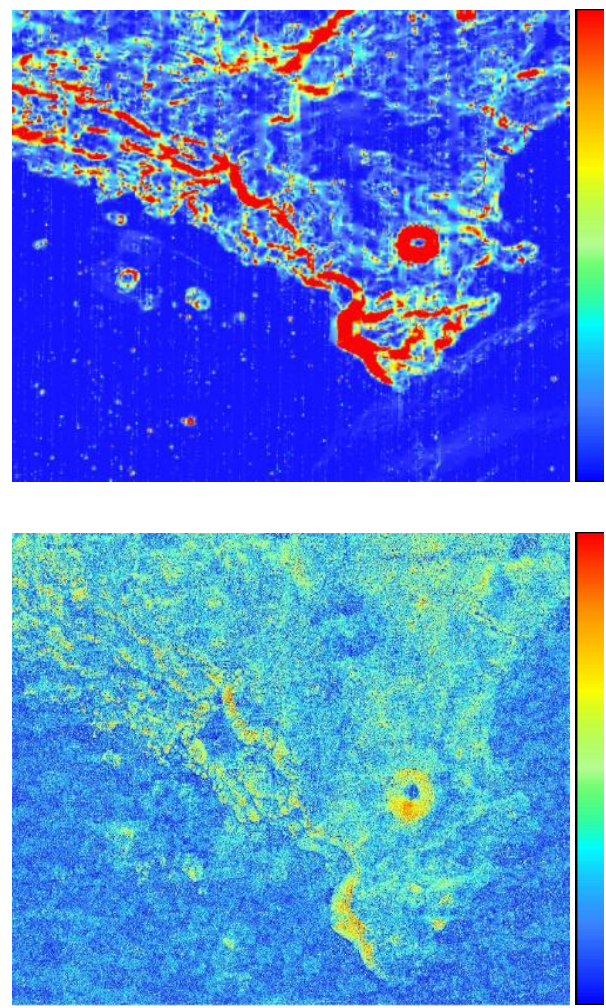

Figure 6. Results by using RMSH (up) and MSR (down) of the tail of Montes Jura southeast

\subsection{Crater Plato}

The distribution of the crater ejecta can be seen apparently in the traditional RMSH image. The bottom, the wall, the edge and the ejecta four parts of the crater can be clearly distinguished. But the ejecta appearance cannot be seen distinctly in MSR image. Because the ejecta material is in cluttered distribution, the overall roughness of the ejecta area is high. The terrain should be gentle at the bottom of the crater, but some high roughness also exists at the bottom of the plain. That indicates that terrain of the crater bottom is not rugged, but the rock surface is rough. Compared with the results obtained by the RMSH, an anomalous region is found in the northern part of the crater on the MSR result. The area is at the edge of the crater, and the roughness value should be similar to the other area edges, but the roughness obtained from the image is extremely low. So that is presumably related to the lithology of the rock at the edge.

The lunar surface minerals possess their own unique diagnostic characteristic absorption bands. By using these bands directly or combining them, the minerals can be identified (Lucey et al, 1995). making use of the spectral data of SELENE, the band 
ratioing is used to reflect the characteristic spectra of the various rocks according to the reflectivity of different rocks (Fischer E M et al, 1996). After several tests, high reflectance of olivine is found at the edge of the crater. Olivine has the glass luster, and its surface is relatively smooth so that the reflectance is high. For this reason, the area is bright on the DOM image. And the roughness based on images using MRS will show the low value on the surface.
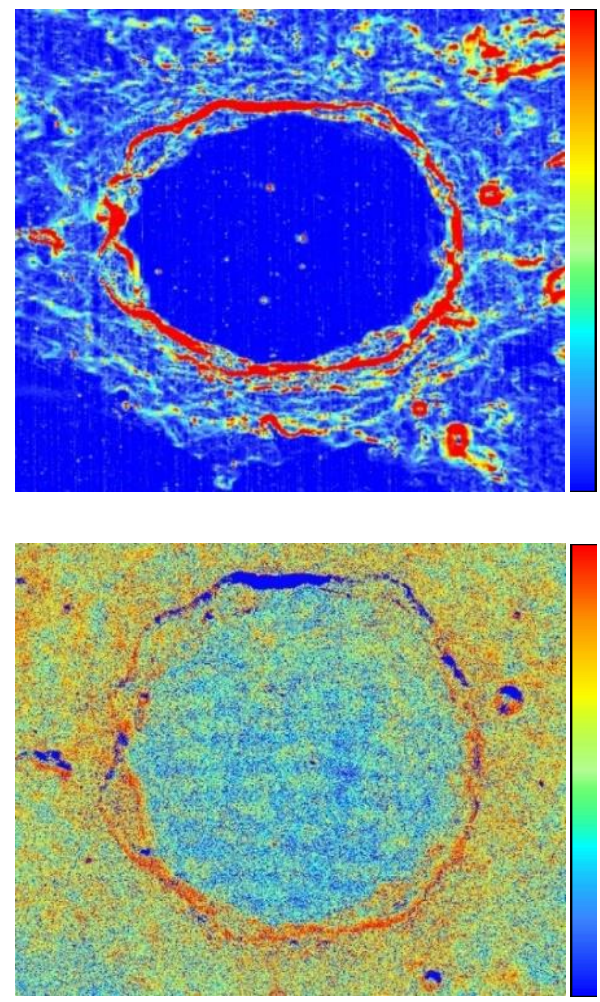

Figure 7. Results by using RMSH (up) and MSR (down) of Crater Plato

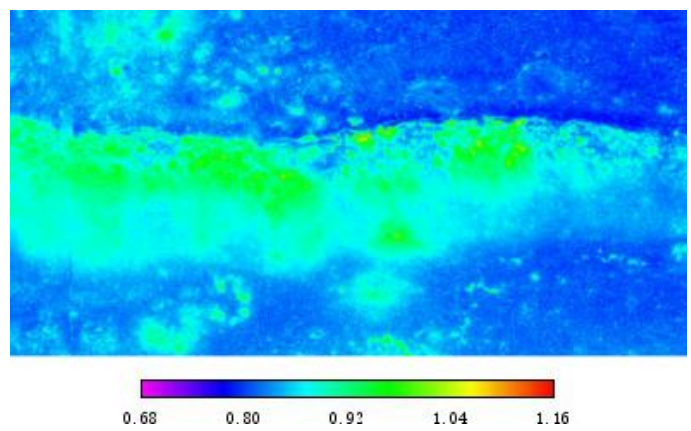

Figure 8. Reflectance spectra of northern cape of Crater Plato edge

\section{CONCLUSIONS}

Data used in this paper including DEM of LRO, CE-2 DOM and the MI data of SELENE. And these data are processed by RMSH, MSR and Band Ratioing respectively. From the results, it is found that the fluctuation largely determines the value of RMSH roughness while lightly effects on the roughness gotten form MSR. The value of the morphological roughness reflects the smooth and rough of the area surface, which is influenced by the lithology of the land largely. Compared with RMSH method, MSR uses a smaller scale. Since MSR calculation is based on the grey-scale image, the illumination becomes one important influencing factor. The reflectivity of different lunar substances is various, so we can gain more information from the MSR results reflected by the illumination.

Based on the above conclusions, we can use the roughness to select the probe's suitable landing points. According to the surface fluctuation situation derived from RMSH, the landing area range can be settled. This can ensure that the lunar probe lands are in a gentle area. And then use the morphological results of the surface smoothness to determine whether the roughness of the landing area is suitable for the detector walking and observing. The combination of the two results provides a new way for selecting future planetary probe landing points.

\section{REFERENCES}

Cheng S, Meng Z, Cui T, et al., 2010. Remote Sensing Geological Analysis of Lunar Satellite in Sinus Iridum[J]. Scientia Sinica Phys, Mech \& Astron, 40(11), pp.13701379 .

Fischer E M, Pieters C M., 1996. Composition and exposure age of the Apollo 16 Cayley and Descartes regions from Clementine data: Normalizing the optical effects of space weathering. Journal of Geophysical Research, 101(E1), pp.2225-2234.

John W M G, David Schleicher, 1972. Geologic atlas of the moon, Plato quadrangle. Department of the interior United States Geological Survey, I-701 LAC-12.

Jolliff B L, Gaddis L R, Ryder G, et al., 2006. New Views of the Moon : Reviews in Mineralogy and Geochemistry. Washington: Mineralogical Society of America.

Lucey, P.G., G.J. Taylor, and F Malaret, 1995. Abundance and distribution of iron on the Moon. Science, 268, pp.11501153.

Orosei R, Bianchi R, Coradini A, et al., 2003. Self - affine behavior of Martian topography at kilometer scale from Mars Orbiter Laser Altimeter data. Journal of Geophysical Research Atmospheres, 108(4).

P.Soille, 2013. Morphological image analysis: principles and applications. Springer Science \& Business Media.

Rosenburg, M.A., Aharonson, O., Head, J.W., Kreslavsky, M.A., Mazarico, E., Neumann, G.A., Smith, D.E., Torrence, M.H., Zuber, M.T., 2011. Global surface slopes and roughness of the Moon from the lunar orbiter laser altimeter. Journal of Geophysical Research, 116, E02001

Shepard M K, Campbell B A, Bulmer M H, et al., 2001. The roughness of natural terrain: A planetary and remote sensing perspective. Journal of Geophysical Research, 106(E12), pp.32777-32795.

Smith, D.E, Zuber, M., Jackson, G., Cavanaugh, J, et al., 2010a. The Lunar Orbiter Laser Altimeter investigation on the Lunar Reconnaissance Orbiter mission. Space Science Reviews. 150, pp.209-241.

Smith, D.E., Zuber, M.T., Neumann, G.A., Lemoine, et al., 
2010b. Initial observations from the Lunar Orbiter Laser Altimeter (LOLA). Geophysical Research Letters, 37(18), pp.109-118.

USGS. 2008. 1:1 Million-Scale Maps of the Moon. United States Geological Survey, LAC11-LAC12.

W. Cao, Z. Cai, and Z. Tang, 2014. Lunar surface roughness based on multiscale morphological method. Planetary and Space Science, 108, pp. 13-2.

W. Gonzalez and R. E. Woods, 2013. Digital image processing using matlab (second edition). Journal of Applied Sciences, 8, pp. 956-965.

Xi Xiaoxu, Wu Zhiyuan, et al., 2012. The Interpretation of the Land Form of Sinus Iridum on the Moon Based on the Roughness. Remote Sensing for Land \& Resources, 24(1), pp.95-99.

Yan Y, Tang G, Xiong L, et al., 2014. Lunar surface roughness of Mare Imbrium based on DEMs. Geographical Research, 33(8), pp.1442-1456.

Ye J, Huang J, Zhang T, et al., 2013. The Achievements of CE-2 Satellite Technology and the Prospect of China 's Deep Space Exploration. Science China Technological Sciences, (5), pp.467-477.

Yokota Y, Haruyama J, Honda C, et al., 2008. Lunar topography: Statistical analysis of roughness on a kilometer scale. Advances in Space Research, 42(2), pp.259-266. 